\title{
The offset-midpoint traveltime pyramid of P-waves in homogeneous orthorhombic media
}

\author{
Qi Hao ${ }^{1}$, Alexey Stovas ${ }^{1}$, and Tariq Alkhalifah ${ }^{2}$
}

\begin{abstract}
The offset-midpoint traveltime pyramid describes the diffraction traveltime of a point diffractor in homogeneous media. We have developed an analytic approximation for the P-wave offset-midpoint traveltime pyramid for homogeneous orthorhombic media. In this approximation, a perturbation method and the Shanks transform were implemented to derive the analytic expressions for the horizontal slowness components of $\mathrm{P}$-waves in orthorhombic media. Numerical examples were shown to analyze the proposed traveltime pyramid formula and determined its accuracy and the application in calculating migration isochrones and reflection traveltime. The proposed offset-midpoint traveltime formula is useful for Kirchhoff prestack time migration and migration velocity analysis for orthorhombic media.
\end{abstract}

\section{INTRODUCTION}

Time migration is a standard step of seismic data processing. The phase-shift migration is often used to handle the poststack and prestack data from vertically heterogeneous models. For the prestack phase-shift migration of common-midpoint gathers, the phase-shift function is constructed by a double-square-root equation in terms of the wavenumber (slowness) components defined in midpoint-offset domain (Yilmaz [2001], p. 638). The multiple integrals in phaseshift migration can be treated by the stationary phase method. Alkhalifah (2000a, 2000b) uses the stationary phase method to obtain an asymptotic solution of the multiple integral of an oscillatory function in the prestack phase-shift offset-midpoint migration for transversely isotropic media with a vertical symmetry axis (VTI). In the oscillatory function, the P-wave traveltime at the stationary point is described by an offset-midpoint traveltime equation, which is also called the offset-midpoint traveltime pyramid or Cheops' pyramid (Claerbout [1985], pp. 164-166; Alkhalifah, 2000a) because of the shape of the offset-midpoint traveltime surface. This treatment of the phase-shift migration leads to the Kirchhoff prestack migration using straight rays, which is extremely efficient for time-domain migration of prestack seismic data, and subsequent migration-based velocity analysis.

The offset-midpoint traveltime pyramid controls the phase term in the Kirchhoff prestack migration based on straight rays. For isotropic media, the P-wave offset-midpoint traveltime pyramid is expressed by a very simple analytic equation (Claerbout [1985], p. 163). For general anisotropic media, it is impossible to find exact and analytic expressions for the offset-midpoint traveltime pyramid of P-waves. Even for VTI media, one cannot find such analytic expression because there exists no exact and analytic relation between phase and group velocities. Although numerical methods for solving inverse problems may accurately obtain the P-wave offsetmidpoint traveltime pyramid for general anisotropic media, this significantly decreases the efficiency of the Kirchhoff prestack time migration. A common way to overcome this problem is to find approximate analytic solutions using perturbation theory. In this way, an approximate traveltime formula can be found for the complex medium under consideration by seeking a small perturbation from an analytic solution valid for a simpler reference medium. For instance, the traveltime of body waves in weakly anisotropic media can be calculated along the reference ray in an isotropic background, in which the weak anisotropy parameters are taken as the perturbation parameters (Farra and Psencik, 2013a, 2013b); the eikonal equation for transversely isotropic media can also be solved by perturbation approaches, and the anellipticity parameter plays the role of perturbation parameter (Stovas and Alkhalifah, 2012, 2013). Recently, we applied a similar perturbation technique to obtain the analytic formulas for offset-midpoint traveltime pyra-

First presented at the EAGE 77th Annual Meeting. Manuscript received by the Editor 30 June 2015; revised manuscript received 26 March 2016; published online 18 July 2016.

${ }^{1}$ Norwegian University of Science and Technology (NTNU), Department of Petroleum Engineering and Applied Geophysics, Trondheim, Norway. E-mail: qi. hao@ntnu.no; alexey.stovas@ntnu.no.

${ }^{2}$ King Abdullah University for Science and Technology (KAUST), Physical Science Division, Thuwal, Saudi Arabia. E-mail: tariq.alkhalifah@kaust.edu.sa.

(C) 2016 Society of Exploration Geophysicists. All rights reserved. 
mids of P-waves in tilted transversely isotropic media (Hao and Stovas, 2014, 2015) and the horizontal transversely isotropic media (Hao et al., 2015).

Orthorhombic anisotropy is characterized by three mutually orthogonal planes of mirror symmetry (Tsvankin [2001], pp. 1011). Orthorhombic symmetry provides a more realistic description of sedimentary basins with parallel vertical fractures or isotropic media with two orthogonal sets of vertical fractures (Schoenberg and Helbig, 1997; Bakulin et al., 2000). An elastic orthorhombic medium includes nine independent stiffness coefficients and three mutually orthogonal planes of mirror symmetry. In each symmetry plane, the medium exhibits transverse isotropy. In this kind of media, the P-wave phase and group velocities are exactly characterized by all nine independent stiffness coefficients. Tsvankin's (1997) notation is widely used to parameterize elastic orthorhombic media. Because the P-wave traveltime and velocity are insensitive to the velocity parameter of S-waves in Tsvankin's (1997) notation, the acoustic approximation (Alkhalifah, 2003) provides a practical assumption for seismic modeling and inversion of P-wave kinematics (e.g., velocity and traveltime) for orthorhombic media. For such models, the $\mathrm{S}$-wave velocities along the three symmetry axes are assumed to be zero. Only six independent parameters are required to describe the P-wave phase and group velocities in this kind of media. Alkhalifah's (2003) notation is normally used to parameterize the acoustic orthorhombic media.

In this paper, we present the P-wave offset-midpoint traveltime pyramid for homogeneous orthorhombic media. We modify Alkhalifah's (2003) notation to characterize P-wave slowness surface for orthorhombic media by replacing the Thomsen (1986)-type parameter $\delta_{3}$ by the corresponding anellipticity parameter. From the prestack phase-shift migration operator for offset-midpoint gathers, the P-wave traveltime at the stationary point is derived by the combination of perturbation theory and the Shanks transform (Bender and Orszag [1978], pp. 369-375). Simple numerical examples are given to analyze the traveltime pyramid formula and to show its applications in generating the migration isochrones and calculating the P-wave reflection traveltime. Applying the offset-midpoint traveltime pyramid to the Kirchhoff prestack time migration for orthorhombic media is explained in the "Discussion" section.

\section{KIRCHHOFF PRESTACK TIME MIGRATION}

The single-trace response of the 3D prestack time-domain phaseshift migration defined in the half-offset-midpoint domain for homogeneous anisotropic media reads (Hao et al., 2015; Appendix A)

$$
\begin{aligned}
& P\left(x_{1}, x_{2}, h_{1}=0, h_{2}=0, \tau, t=0\right) \\
& \quad=\int_{-\infty}^{\infty} \int_{-\infty}^{\infty} \int_{-\infty}^{\infty} \int_{-\infty}^{\infty} \int_{-\infty}^{\infty} \tilde{P}\left(x_{1}^{0}, x_{2}^{0}, h_{1}^{0}, h_{2}^{0}, \tau=0, \omega\right) \\
& \quad \times \exp (i \omega T) d \omega d k_{h 1} d k_{h 2} d k_{x 1} d k_{x 2},
\end{aligned}
$$

where subscripts 1 and 2 denote the $x$-and $y$-axes of an acquisition system, respectively; $t$ denotes the time; $\omega$ denotes the angular frequency; $P\left(x_{1}, x_{2}, h_{1}, h_{2}, \tau, t\right)$ denotes the time-domain commonmidpoint data extrapolated to the "time depth" $\tau$, where the time depth denoting the two-way traveltime of vertically propagating $\mathrm{P}$-waves is used to describe the extrapolation depth in time domain; $\left(x_{1}, x_{2}\right)$ denotes the midpoint position; $\left(h_{1}, h_{2}\right)$ denotes the source- receiver half-offset; $\left(x_{1}, x_{2}, z=\tau v_{p 0} / 2\right)$ denotes the position of an image point in depth domain, where $v_{p 0}$ denotes the vertical velocity of P-waves; $P\left(x_{1}, x_{2}, h_{1}=0, h_{2}=0, \tau, t=0\right)$ denotes the seismic image after the prestack time migration using the exploring reflector imaging condition; $\tilde{P}\left(x_{1}^{0}, x_{2}^{0}, h_{1}^{0}, h_{2}^{0}, \tau=0, \omega\right)$ denotes the frequencydomain single-trace surface seismic data, where the position of the single trace in data space is described by the midpoint position $\left(x_{1}^{0}, x_{2}^{0}\right)$ and the source-receiver half-offset $\left(h_{1}^{0}, h_{2}^{0}\right) ;\left(k_{x 1}, k_{x 2}\right)$ and $\left(k_{h 1}, k_{h 2}\right)$ denote the horizontal wavenumber vectors of midpoint and source-receiver half-offset; and $T$ is the traveltime shift that is expressed by

$$
\begin{aligned}
T\left(p_{s 1}, p_{s 2}, p_{g 1}, p_{g 2}\right)= & \frac{1}{2}\left(q_{s}+q_{g}\right) \tau v_{p 0}+p_{s 1} y_{s 1} \\
& +p_{s 2} y_{s 2}+p_{g 1} y_{g 1}+p_{g 2} y_{g 2},
\end{aligned}
$$

where $\left(y_{s 1}, y_{s 2}\right)=\left(x_{1}^{0}-h_{1}^{0}-x_{1}, x_{2}^{0}-h_{2}^{0}-x_{2}\right)$ denotes the lateral distance vector between the image point and the source; $\left(y_{g 1}, y_{g 2}\right)=$ $\left(x_{1}^{0}+h_{1}^{0}-x_{1}, x_{2}^{0}+h_{2}^{0}-x_{2}\right)$ denotes the lateral distance vector between the image point and the receiver; $q_{s}$ and $q_{g}$ are the vertical slowness components of source and receiver, which are the functions of the horizontal slowness components; $\left(p_{s 1}, p_{s 2}\right)$ and $\left(p_{g 1}, p_{g 2}\right)$ are the horizontal slowness vectors of source and receiver, which are linearly linked to the half-offset slowness vector $\left(p_{h 1}, p_{h 2}\right)=\left(k_{h 1} /(2 \omega), k_{h 2} /(2 \omega)\right)$ and the midpoint slowness vector $\left(p_{x 1}, p_{x 2}\right)=\left(k_{x 1} /(2 \omega), k_{x 2} /(2 \omega)\right)$ by the following relationship:

$$
\begin{aligned}
& p_{s 1}=p_{x 1}-p_{h 1}, \\
& p_{s 2}=p_{x 2}-p_{h 2}, \\
& p_{g 1}=p_{x 1}+p_{h 1}, \\
& p_{g 2}=p_{x 2}+p_{h 2} .
\end{aligned}
$$

Equation 2 represents the traveltime of the plane wave propagating from the source to the image point and back to the receiver. The geometric explanation of these quantities in equations 1 and 2 is shown in Figure 1.

The integrals in equation 1 can be estimated by the stationary phase method. The stationary point of the phase function in equation 1 corresponds to the local minimum or maximum of the traveltime shift 2. Hence, the stationary point is found from the equations

$$
\frac{\partial q_{(s, g)}}{\partial p_{i(s, g)}}=-\frac{2 y_{i(s, g)}}{\tau v_{p 0}}, \quad i=1,2,
$$

where the vector $\left(p_{1}, p_{2}\right), q$, and $\left(y_{1}, y_{2}\right)$ are either $\left(p_{s 1}, p_{s 2}\right), q_{s}$, and $\left(y_{s 1}, y_{s 2}\right)$ for the source or $\left(p_{g 1}, p_{g 2}\right), q_{g}$, and $\left(y_{g 1}, y_{g 2}\right)$ for the receiver, respectively. Traveltime shift 2 with stationary phase condition 4 describes the exact traveltime of seismic ray from the source to the image point then back to the receiver. Using the stationary phase method, we finally derive the closed-form expression for equation 1 (Appendix A):

$$
\begin{aligned}
& P\left(x_{1}, x_{2}, h_{1}=0, h_{2}=0, \tau, t=0\right) \\
& \quad \approx \frac{1}{4 \pi^{2} \sqrt{\left|\lambda_{s} \lambda_{g}\right|}} \frac{\partial^{2}}{\partial \tilde{T}^{2}} \tilde{P}\left(x_{1}^{0}, x_{2}^{0}, h_{1}^{0}, h_{2}^{0}, \tau=0, \tilde{T}\right) \cos \xi
\end{aligned}
$$


which is the single-trace response of the Kirchhoff prestack time migration based on straight rays. Here, $\tilde{P}\left(x_{1}^{0}, x_{2}^{0}, h_{1}^{0}, h_{2}^{0}, \tau=0, t\right)$ denotes the time-domain single-trace surface seismic data with midpoint $\left(x_{1}^{0}, x_{2}^{0}\right)$ and source-receiver half-offset $\left(h_{1}^{0}, h_{2}^{0}\right) ; \tilde{T}$ is the diffraction traveltime of P-wave rays, $\tilde{T}=T\left(\tilde{p}_{s 1}, \tilde{p}_{s 2}, \tilde{p}_{g 1}, \tilde{p}_{g 2}\right)$, corresponding to traveltime shift 2 under stationary phase condition 4 , where $\left(\tilde{p}_{s 1}, \tilde{p}_{s 2}\right)$ and $\left(\tilde{p}_{g 1}, \tilde{p}_{g 2}\right)$ are the horizontal slowness vectors of source and receiver at stationary point; $\lambda_{s}$ and $\lambda_{g}$ are linked to the Gaussian curvatures of the slowness surfaces of the source and receiver; $\xi$ is the phase shift depending on the signs of principal curvatures of the slowness surfaces of source and receiver. All these quantities are explicitly expressed in terms of the horizontal slowness components of source and receiver. However, it is generally complex to calculate source and receiver slownesses from midpoint and source-receiver offset. In the following two sections, we derive analytic approximations for horizontal slowness components and traveltimes at the stationary points. Because the derivation is valid for source and receiver, we use symbol $\left(p_{1}, p_{2}\right)$ to represent the horizontal slowness vectors of either source or receiver. In addition, in the following sections (apart from Appendix A), the horizontal slowness components $p_{1}$ and $p_{2}$ and the traveltime $T$ stand for their values at the stationary point (the tildes above $\left(p_{1}, p_{2}\right)$ and $T$ are neglected for convenience).

\section{SLOWNESS APPROXIMATION AT STATIONARY POINT}

Under the acoustic assumption (Alkhalifah, 1998, 2003), the phase and group velocities of P-waves in a homogeneous orthorhombic medium can be normally characterized by Alkhalifah's (2003) notation including the P-wave vertical velocity $v_{p 0}$; the normal moveout (NMO) velocity $v_{n 2}$ and the anellipticity parameter $\eta_{2} \equiv\left(\varepsilon_{2}-\delta_{2}\right) /\left(1+2 \delta_{2}\right)$ defined in the vertical symmetry $[x, z]$ plane; the NMO velocity $v_{n 1}$ and the anellipticity parameter $\eta_{1} \equiv\left(\varepsilon_{1}-\delta_{1}\right) /\left(1+2 \delta_{1}\right)$ defined in the vertical symmetry $[y, z]$ plane; and the anisotropy parameter $\delta_{3}$ defined in the horizontal symmetry $[x, y]$ plane. We slightly modify Alkhalifah's (2003) notation by introducing the anellipticity parameter $\eta_{3} \equiv\left(\varepsilon_{1}-\varepsilon_{2}-\right.$ $\left.\delta_{3}\left(1+2 \varepsilon_{2}\right)\right) /\left(\left(1+2 \delta_{3}\right)\left(1+2 \varepsilon_{2}\right)\right.$ ) (Vasconcelos and Tsvankin, 2006) defined in the $[x, y]$ plane instead of the anisotropy parameter $\delta_{3}$ (Appendix B). The similar modifications on Alkhalifah's (2003) notation may also be seen in Hao and Stovas (2016a, 2016b) and Stovas (2015). As a result, the P-wave vertical slowness component $q$ is written as a function of the horizontal slowness components $p_{1}$ and $p_{2}$ :

$$
q^{2}=\frac{1}{v_{p 0}^{2}\left(1+2 \eta_{3}\right)} \frac{f_{1}\left(p_{1}, p_{2}\right)}{f_{2}\left(p_{1}, p_{2}\right)}
$$

where

$$
\begin{aligned}
f_{1}\left(p_{1}, p_{2}\right)= & 1-\left(1+2 \eta_{2}\right) v_{n 2}^{2} p_{1}^{2}-\left(1+2 \eta_{1}\right) v_{n 1}^{2} p_{2}^{2} \\
& +2 \eta_{3}\left(1-\left(1+2 \eta_{2}\right) v_{n 2}^{2} p_{1}^{2}\right)\left(1-\left(1+2 \eta_{1}\right) v_{n 1}^{2} p_{2}^{2}\right),
\end{aligned}
$$

$$
f_{2}\left(p_{1}, p_{2}\right)=1-2 \eta_{2} v_{n 2}^{2} p_{1}^{2}-2 \eta_{1} v_{n 1}^{2} p_{2}^{2}-2 \Omega v_{n 1}^{2} v_{n 2}^{2} p_{1}^{2} p_{2}^{2},
$$

with

$$
\begin{aligned}
\Omega= & \left(1+\eta_{1}+\eta_{2}+\eta_{3}-\sqrt{1+2 \eta_{1}} \sqrt{1+2 \eta_{2}} \sqrt{1+2 \eta_{3}}\right. \\
& \left.-4 \eta_{1} \eta_{2} \eta_{3}\right) /\left(1+2 \eta_{3}\right) .
\end{aligned}
$$

It is worth mentioning that Stovas (2015) defines a new parameter $\eta_{x y}$ and replaces the anellipticity parameter $\eta_{3}$ to describe the slowness surface equation for acoustic orthorhombic media. From equations 4 to 9 , we can eliminate the vertical slowness component $q$ and obtain two eighth-order algebraic equations in terms of $p_{1}^{2}$ and $p_{2}^{2}$ (see equations $\mathrm{C}-1$ and $\mathrm{C}-2$ in Appendix C). Because there is no exact and analytic formula to express the exact solution to these equations, we seek their approximate solution by defining the following perturbation expansions with respect to the three anellipticity parameters,

$$
\begin{aligned}
& p_{1}^{2}=c_{0}+\sum_{i=1}^{3} c_{i} \eta_{i}+\sum_{i, j=1, i \leq j}^{3} c_{i j} \eta_{i} \eta_{j}, \\
& p_{2}^{2}=d_{0}+\sum_{i=1}^{3} d_{i} \eta_{i}+\sum_{i, j=1, i \leq j}^{3} d_{i j} \eta_{i} \eta_{j},
\end{aligned}
$$

where $c_{0}, c_{i}, c_{i j}, d_{0}, d_{i}$, and $d_{i j}$ are the undetermined coefficients. All these coefficients are determined in Appendix $\mathrm{C}$ by substituting expansions 10 and 11 into the two algebraic equations (see equations C-1 and C-2 in Appendix C). For a given pair of midpoint and half-offset, equations 10 and 11 require the NMO velocities $\left(v_{n 1}\right.$ and $\left.v_{n 2}\right)$, the three anellipticity parameters $\left(\eta_{1}, \eta_{2}\right.$, and $\left.\eta_{3}\right)$, and the two-

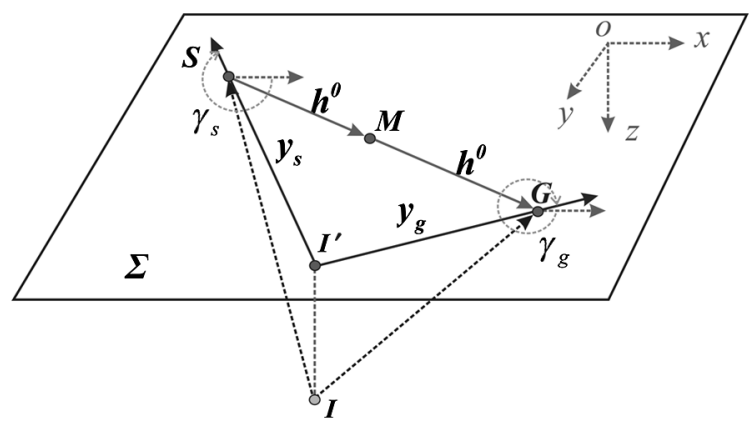

Figure 1. The geometric explanation of quantities in equations 1 and 2 (after Hao et al., 2015). In the Cartesian coordinate system (oxyz), the source $\boldsymbol{S}=\left(s_{1}, s_{2}, 0\right)$, the receiver $\boldsymbol{G}=\left(g_{1}, g_{2}, 0\right)$, and the midpoint $\boldsymbol{M}=\left(x_{1}^{0}, x_{2}^{0}, 0\right)$ are located on the surface $\boldsymbol{\Sigma}$. The halfoffset vector $\boldsymbol{h}^{0}=\left(h_{1}^{0}, h_{2}^{0}, 0\right)$ equals a half of the distance vector from the source $\boldsymbol{S}$ to the receiver $\boldsymbol{G}$. The point $\boldsymbol{I}=\left(x_{1}, x_{2}, z\right)$ is an image point. The point $\boldsymbol{I}^{\prime}=\left(x_{1}, x_{2}, 0\right)$ is the projection of the image point $\boldsymbol{I}$; on the surface $\boldsymbol{\Sigma}$; . The vector $\boldsymbol{y}_{s}=\left(y_{s 1}, y_{s 2}\right)$ denotes the lateral distance between the source $S$ and the image point $I$, and the vector $\boldsymbol{y}_{g}=\left(y_{g 1}, y_{g 2}\right)$ denotes the lateral distance between the receiver $\boldsymbol{G}$ and the image point $\boldsymbol{I}$. The values $\gamma_{s}$ and $\gamma_{g}$ denote the azimuths of vectors $\boldsymbol{y}_{s}$ and $\boldsymbol{y}_{g}$, respectively. The plane wave from the image point $\boldsymbol{I}$ to the source $\boldsymbol{S}$ has the source slowness $\left(p_{s 1}, p_{s 2},-q_{s}\right)$, and the plane wave from the image point $\boldsymbol{I}$ to the receiver $\boldsymbol{G}$ has the receiver slowness $\left(p_{g 1}, p_{g 2},-q_{g}\right)$. These slownesses in the source-receiver domain may be converted to the midpoint-half-offset domain through equation 3 . 
way traveltime $\tau$ of vertically propagating P-waves in an orthorhombic medium. It is worth mentioning that equations 10 and 11 are independent of the velocity $v_{p 0}$ of vertically propagating P-waves.

Then, we try to improve the accuracy of approximation for the horizontal slowness component. The magnitude of the horizontal slowness squared $p^{2}$ is obtained by taking the sum of equations 10 and 11. We use the Shanks transformation (Bender and Orszag [1978], pp. 369-375) to accelerate the convergence of the expansion for $p^{2}$. Consequently, we find

$$
p^{2}=G_{0}+\frac{G_{1}^{2}}{G_{1}-G_{2}},
$$

where

$$
\begin{aligned}
G_{0} & =c_{0}+d_{0}, \\
G_{1} & =\sum_{i}^{3}\left(c_{i}+d_{i}\right) \eta_{i}, \\
G_{2} & =\sum_{i, j=1, i \leq j}^{3}\left(c_{i j}+d_{i j}\right) \eta_{i} \eta_{j} .
\end{aligned}
$$

To further calculate the values of $p_{1}$ and $p_{2}$, we define a nonphysical azimuth $\alpha$ as $\alpha=\arctan \left(p_{1}^{2} / p_{2}^{2}\right)$, which denotes the azimuth of vector $\left(p_{1}^{2}, p_{2}^{2}\right)$ measured from the $x$-axis. From equations 10 and 11 , we find the azimuth $\alpha$ given by

$$
\tan \alpha=\frac{c_{0}+\sum_{i=1}^{3} c_{i} \eta_{i}+\sum_{i, j=1, i \leq j}^{3} c_{i j} \eta_{i} \eta_{j}}{d_{0}+\sum_{i=1}^{3} d_{i} \eta_{i}+\sum_{i, j=1, i \leq j}^{3} d_{i j} \eta_{i} \eta_{j}}, \quad \alpha \in[0, \pi / 2]
$$

From these operations, it follows that the horizontal slowness components are given by

$$
\begin{aligned}
& p_{1}=\operatorname{sgn}\left(y_{1}\right) p \sqrt{\cos \alpha}, \\
& p_{2}=\operatorname{sgn}\left(y_{2}\right) p \sqrt{\sin \alpha} .
\end{aligned}
$$

In equations 15 and 16 , the operator $\operatorname{sgn}\left(y_{i}\right)(i=1,2)$ makes sure that the horizontal slowness component $p_{i}(i=1,2)$ and the horizontal projection of propagation distance $y_{i}(i=1,2)$ always have the same sign. These conditions preserve the horizontal projections of the slowness vector and the ray velocity vector being in the same quadrant of the $[x, y]$ plane.

\section{OFFSET-MIDPOINT TRAVELTIME PYRAMID}

For the given midpoint and source-receiver half-offset, explicit expressions for the horizontal slowness components of source and receiver were derived in the previous section. Therefore, substituting the slowness surface equation 6 , the diffraction traveltime 2 under the stationary phase condition 4 is written as an analytic function of the midpoint and source-receiver half-offset

$$
\begin{aligned}
& T\left(x_{1}, x_{2}, x_{1}^{0}, x_{2}^{0}, h_{1}^{0}, h_{2}^{0}, \tau\right) \\
& \quad=\frac{\tau}{2 \sqrt{1+2 \eta_{3}}}\left(\sqrt{\frac{f_{1}\left(p_{s 1}, p_{s 2}\right)}{f_{2}\left(p_{s 1}, p_{s 2}\right)}}+\sqrt{\frac{f_{1}\left(p_{g 1}, p_{g 2}\right)}{f_{2}\left(p_{g 1}, p_{g 2}\right)}}\right) \\
& \quad+p_{s 1} y_{s 1}+p_{s 2} y_{s 2}+p_{g 1} y_{g 1}+p_{g 2} y_{g 2} .
\end{aligned}
$$

This formula is aimed to calculate the traveltime $T$ in the expression for the single-trace response of the Kirchhoff prestack time migration (equation 5). Because $T$ is entirely dependent on the midpoint and the source-receiver half-offset for a single point diffractor $\left(x_{1}, x_{2}, z=v_{p 0} \tau / 2\right)$, formula 17 is referred as the offset-midpoint traveltime pyramid for orthorhombic media. For a single diffractor $\left(x_{1}, x_{2}, z=v_{p 0} \tau / 2\right)$ in a homogeneous orthorhombic medium, the diffraction traveltime corresponding to the midpoint $\left(x_{1}^{0}, x_{2}^{0}\right)$ and the half-offset $\left(h_{1}^{0}, h_{2}^{0}\right)$ is calculated in equation 17 , where the horizontal slowness components $\left(p_{s 1}, p_{s 2}\right)$ for source and $\left(p_{g 1}, p_{g 2}\right)$ for receiver are calculated from equations 12 to 16; the vector $\left(y_{s 1}, y_{s 2}\right)$ between source and midpoint and the vector $\left(y_{g 1}, y_{g 2}\right)$ between receiver and midpoint are defined after equation 2 . As explained within the text after equation 11, for a given pair of midpoint and half-offset, calculating the horizontal slowness components in formula 17 only requires the NMO velocities $\left(v_{n 1}\right.$ and $\left.v_{n 2}\right)$, the three anellipticity parameters $\left(\eta_{1}, \eta_{2}\right.$, and $\left.\eta_{3}\right)$, and the twoway traveltime $\tau$ of vertically propagating P-waves in an orthorhombic medium. Therefore, formula 17 is independent of the velocity $v_{p 0}$ of vertically propagating P-waves.

Until now, we discuss only the case of orthorhombic media with three symmetry planes orthogonal to the axes of acquisition system. To apply the traveltime equation 17 for azimuthal orthorhombic media, the lateral coordinate rotations are required. We assume the lateral position of image point, midpoint and source-receiver half-offsets for such media are denoted by uppercase symbols $\left(X_{1}, X_{2}\right),\left(X_{1}^{0}, X_{2}^{0}\right)$, and $\left(H_{1}^{0}, H_{2}^{0}\right)$. These coordinates need to be transformed to the corresponding lowercase symbols in formula 17. For instance, the source-receiver offset $\left(H_{1}^{0}, H_{2}^{0}\right)$ is projected to $\left(h_{1}^{0}, h_{2}^{0}\right)$ by the following rotation:

$$
\begin{gathered}
h_{1}^{0}=H_{1}^{0} \cos \phi+H_{2}^{0} \sin \phi, \\
h_{2}^{0}=-H_{1}^{0} \sin \phi+H_{2}^{0} \cos \phi,
\end{gathered}
$$

where $\phi$ denotes the azimuth of the $[x, z]$ symmetry plane of an orthorhombic medium in the acquisition system. The functions $\left(X_{1}, X_{2}\right)$ and $\left(X_{1}^{0}, X_{2}^{0}\right)$ can be obtained in a similar way.

\section{TRAVELTIME PYRAMID FOR 2D VTI MEDIA}

The vertical symmetry planes of an orthorhombic medium exhibit transverse isotropy. Therefore, the traveltime pyramid derived in the previous section can be reduced to the traveltime pyramid for homogeneous VTI media. Without loss of generality, we consider the $[x, z]$ plane of a homogeneous VTI medium. In this case, equation 17 is finally reduced to the traveltime pyramid for 2D VTI media (Alkhalifah, 2000b) 


$$
\begin{aligned}
T\left(x, x^{0}, h^{0}, \tau\right)= & \frac{\tau}{2}\left(\sqrt{1-\frac{v_{n}^{2} p_{s}^{2}}{1-2 v_{n}^{2} \eta p_{s}^{2}}}+\sqrt{1-\frac{v_{n}^{2} p_{g}^{2}}{1-2 v_{n}^{2} \eta p_{g}^{2}}}\right) \\
& +p_{s} y_{s}+p_{g} y_{g},
\end{aligned}
$$

where $x$ denotes the lateral position of the diffraction point; $x^{0}$ and $h^{0}$ denote the midpoint and the source-receiver half-offset; $\tau$ denotes the vertical two-way traveltime from the diffraction point to the surface; $v_{n}$ and $\eta$ denote the NMO velocity and the anellipticity parameter for the VTI medium; $y_{s}=x^{0}-h^{0}-x$ and $y_{g}=x^{0}+h^{0}-x$ denote the lateral distances from source and receiver to the diffraction point; and $p_{s}$ and $p_{g}$ denote source and receiver slownesses for a $2 \mathrm{D}$ VTI medium,

$p_{s, g}^{2}=\frac{Y_{s, g}^{2}\left(Y_{s, g}^{6}+6 v_{n}^{2}(1-\eta) \tau^{2} Y_{s, g}^{4}+3 v_{n}^{4}(3+4 \eta) \tau^{4} Y_{s, g}^{2}+4 v_{n}^{6} \tau^{6}\right)}{v_{n}^{2}\left(Y_{s, g}^{2}+v_{n}^{2} \tau^{2}\right)\left((1+2 \eta) Y_{s, g}^{6}+2 v_{n}^{2}(3+5 \eta) \tau^{2} Y_{s, g}^{4}+v_{n}^{4}(9+44 \eta) \tau^{4} Y_{s, g}^{4}+4 v_{n}^{6} \tau^{6}\right)}$,

with

$$
Y_{s, g}=2 y_{s, g} .
$$

Equation 21 is obtained from equation 12 with equations 13 and equations C-9-C-29 in Appendix C, and it is first presented by Alkhalifah (2000b).

\section{NUMERICAL EXAMPLES}

First, we test the accuracy of traveltime formula 17. The relative error in traveltime is measured by the following formula:

$$
E=\left|1-T_{\text {appr }} / T_{\text {exact }}\right| \times 100 \%,
$$

where $T_{\text {appr }}$ denotes the approximate traveltime calculated by equation 17 and $T_{\text {exact }}$ denotes the exact traveltime. The exact traveltime is obtained by the following procedure: (1) to numerically solve the nonlinear equations $\mathrm{C}-1$ and $\mathrm{C}-2$ with equations C-3-C-6 for horizontal slowness components $p_{1}$ and $p_{2}$ for source and receiver, respectively, and (2) to substitute the horizontal slowness components for source and receiver, calculated in the previous step, into the traveltime equation 2 .

Figure 2 shows the relative errors of traveltime pyramid along different acquisition azimuths for a homogeneous, elliptically orthorhombic model. For elliptically orthorhombic media, the three anellipticity parameters are zero. The horizontal slownesses for source and receiver, calculated from equations $\mathrm{C}-1$ and $\mathrm{C}-2$, are exact for elliptically orthorhombic media. In this case, traveltime pyramid 17 is reduced to the exact expression. This is verified in Figure 2, which shows that the relative errors of traveltime pyramid 17 are so small for elliptically orthorhombic media. The small relative errors are due to the numerical algorithm when numerically calculating the exact offset-midpoint traveltime pyramid.

Figure 3 shows the relative errors of traveltime pyramid 17 for a homogeneous orthorhombic model with three nonzero anellipticity parameters. Figure $3 \mathrm{a}$ and $3 \mathrm{~d}$ corresponds to the acquisition azimuths coinciding with the two vertical symmetry planes of the orthorhombic model. The two plots show that the relative errors in traveltime are far smaller than $0.1 \%$, and traveltime pyramid 17 is accurate enough in this case. The explanation to this phenomenon is that only one anellipticity parameter $\left(\eta_{1}\right.$ or $\left.\eta_{2}\right)$ affects the accuracy of traveltime pyramid 17 when the acquisition azimuth coincides with one of the vertical symmetry planes of orthorhombic media. Figure $3 \mathrm{a}$ and $3 \mathrm{~d}$ also proves the high accuracy of the traveltime pyramid formula for VTI media (Alkhalifah, 2000b). For the acquisition azimuth off the vertical symmetry planes of orthorhombic media, however, Figure $3 b$ and $3 c$ illustrates that the relative error of traveltime pyramid 17 increases significantly. This is because all three anellipticity parameters affect the accuracy of traveltime pyramid 17, when the acquisition azimuth does not coincide with the vertical symmetry planes of orthorhombic media. The comparison between Figures 2 and 3 illustrate that the accuracy of traveltime pyramid 17 is significantly affected by the three anellipticity parameters when the acquisition azimuth is off the vertical symmetry planes of an orthorhombic medium.

Figure 4 illustrates the offset-midpoint traveltime for different acquisition azimuths. The peak of diffraction traveltime surfaces correspond to the vertical two-way traveltime from the diffractor to the acquisition surface. It is obvious that NMO velocity parameters and
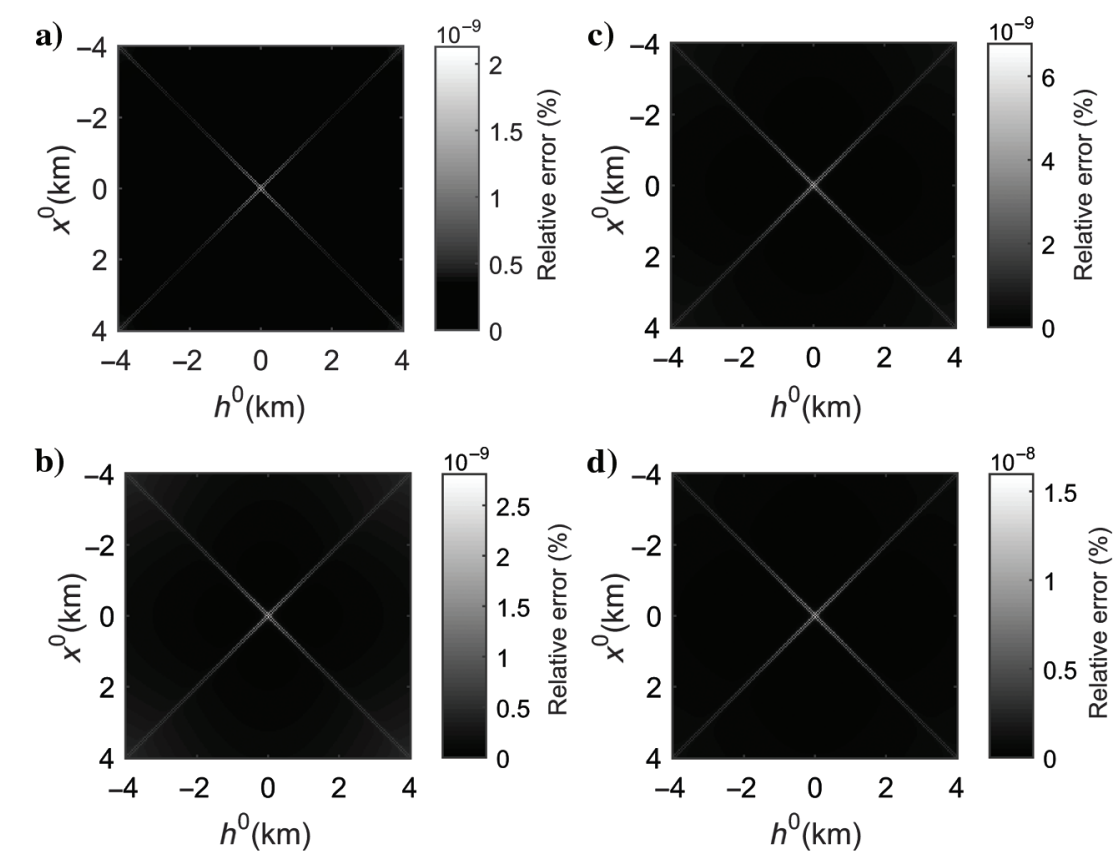

Figure 2. The relative error of the traveltime pyramid formula along the acquisition azimuths (a) 0 , (b) $\pi / 6$, (c) $\pi / 3$, and (d) $\pi / 2$ in an elliptically orthorhombic medium. The functions $x^{0}$ and $h^{0}$ denote the midpoint and offset along acquisition azimuth. The medium parameters include the P-wave vertical velocity $v_{p 0}=3 \mathrm{~km} / \mathrm{s} ; \mathrm{NMO}$ velocities $v_{n 1}=3.5 \mathrm{~km} / \mathrm{s}$ and $v_{n 2}=2.5 \mathrm{~km} / \mathrm{s}$; three anellipticity parameters $\eta_{1}, \eta_{2}$, and $\eta_{3}$ equal to zeros. The single diffraction point is located behind the coordinate origin. The minimum zero-offset two-way traveltime is $\tau=0.667 \mathrm{~s}$. 

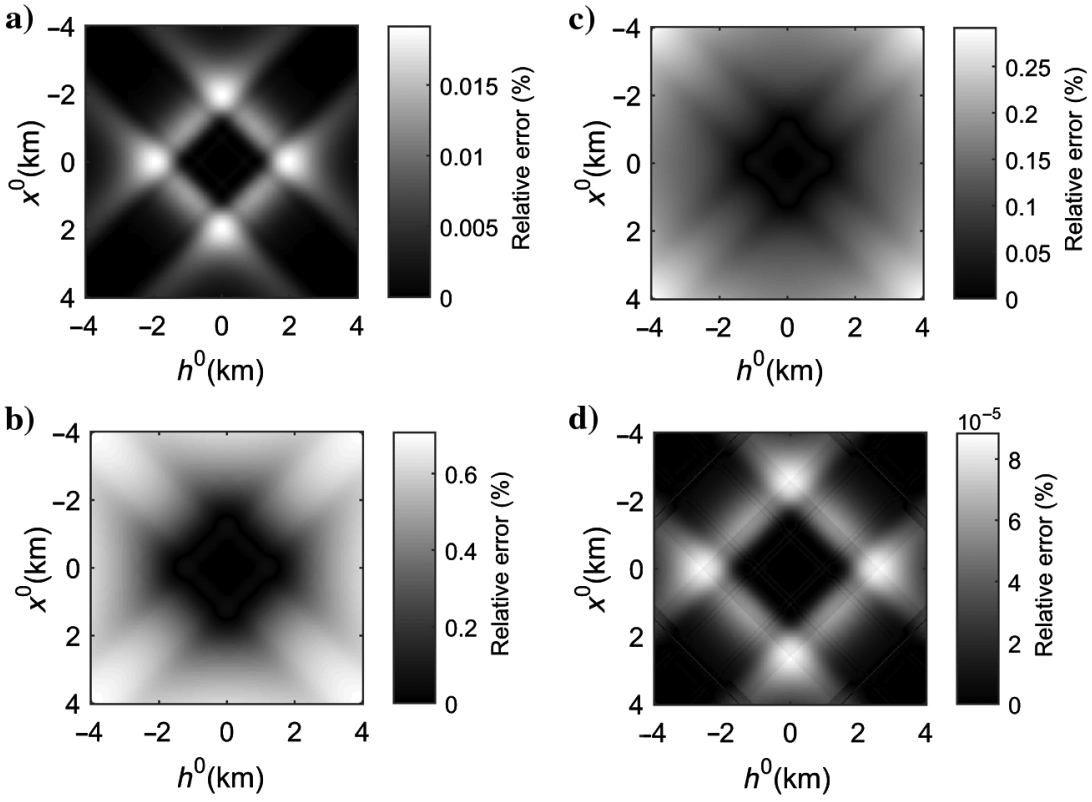

Figure 3. The relative error of the traveltime pyramid formula along the acquisition azimuths (a) 0 , (b) $\pi / 6$, (c) $\pi / 3$, and (d) $\pi / 2$ in an orthorhombic medium. The functions $x^{0}$ and $h^{0}$ denote the midpoint and offset along an acquisition azimuth. The medium parameters include the P-wave vertical velocity $v_{p 0}=3 \mathrm{~km} / \mathrm{s}$; NMO velocities $v_{n 1}=$ $3.5 \mathrm{~km} / \mathrm{s}$ and $v_{n 2}=2.5 \mathrm{~km} / \mathrm{s}$; anellipticity parameters $\eta_{1}=0.1, \eta_{2}=0.3$, and $\eta_{3}=0.2$. The single diffraction point is located behind the coordinate origin. The minimum zero-offset two-way traveltime is $\tau=0.667 \mathrm{~s}$.
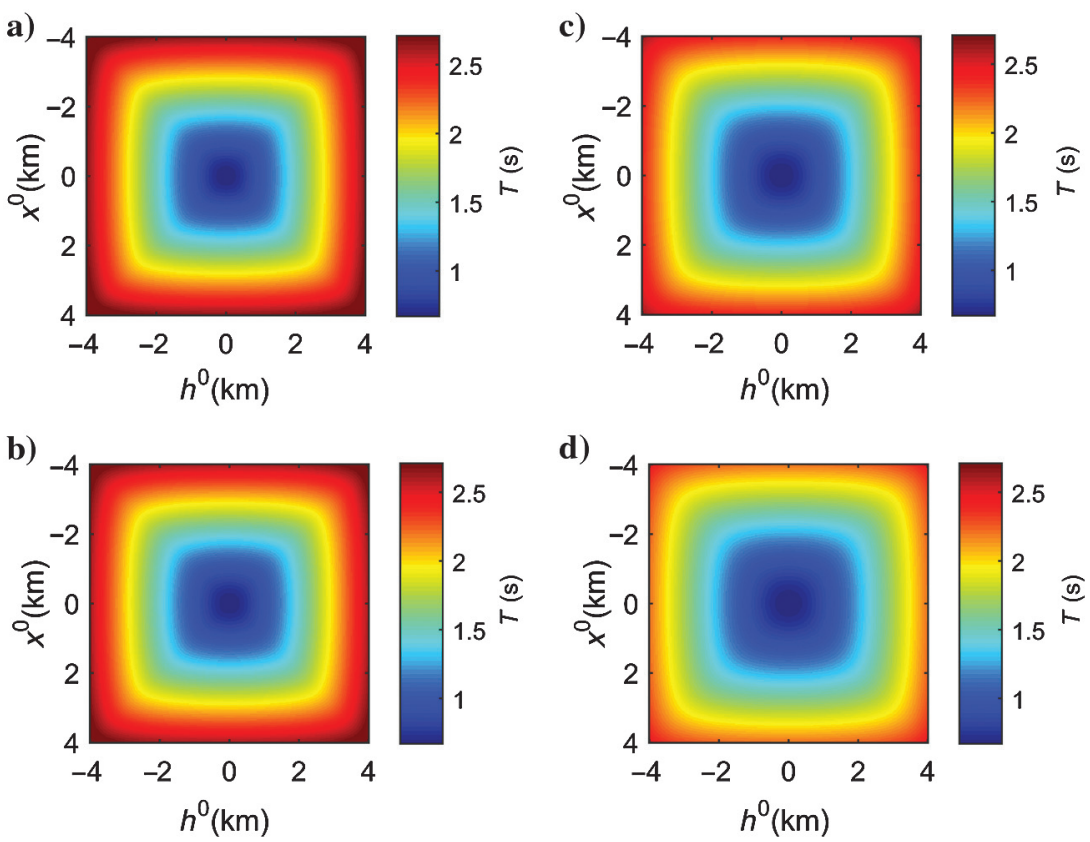

Figure 4. The offset-midpoint traveltime pyramids along the acquisition azimuths (a) 0 , (b) $\pi / 6$, (c) $\pi / 3$, and (d) $\pi / 2$ in an orthorhombic medium. The values $x^{0}$ and $h^{0}$ denote the midpoint and offset along an acquisition azimuth. The medium parameters include the P-wave vertical velocity $v_{p 0}=3 \mathrm{~km} / \mathrm{s} ; \mathrm{NMO}$ velocities $v_{n 1}=3.5 \mathrm{~km} / \mathrm{s}$ and $v_{n 2}=2.5 \mathrm{~km} / \mathrm{s}$; anellipticity parameters $\eta_{1}=0.1, \eta_{2}=0.3$, and $\eta_{3}=0.2$. The single diffraction point is located behind the coordinate origin. The minimum zero-offset twoway traveltime is $\tau=0.667 \mathrm{~s}$. All plots are illustrated in the same color scale. anellipticity parameters affect the shape of traveltime surface. We can see that the offset-midpoint traveltime is anisotropic.

Figure 5 shows the migration isochrones for the common-offset prestack time-domain Kirchhoff's migration using the proposed traveltime formula 17. The migration isochrones are composed of diffractors, which have the same diffraction time for a fixed pair of source and receiver. Each time sampling in a single trace is mapped along the migration isochrones. The seismic image is the diffraction superposition of all mapped data in subsurface. We can see that for the vertical orthorhombic media, the azimuthal variation of migration isochrones happens when the propagation direction of $\mathrm{P}$-waves is away from the vertical direction. This indicates that the anisotropic effect can be obvious in the migration image only for moderate and large offset surface data if we do not consider the lateral variations of models.

The traveltime pyramid 17 can be implemented to calculate the $\mathrm{P}$-wave reflection traveltime in a horizontal orthorhombic layer. Figures 6, 7, 8, and 9 show the accuracy comparison between the traveltime pyramid 17 and the nonhyperbolic moveout approximation (Xu et al., 2005; Vasconcelos and Tsvankin, 2006). This indicates that the traveltime pyramid 17 is more accurate than the nonhyperbolic moveout approximation for orthorhombic media with weak to strong anisotropy. As we note in the second example, the three anellipticity parameters significantly affect the accuracy of the reflection traveltime approximation. For relatively small values of anellipticity parameters, the traveltime formula 17 is accurate enough. However, the accuracy obviously decreases when increasing the values of anellipticity parameters. This means that the proposed traveltime approximation is valid for orthorhombic media with weak to moderate anellipticity.

\section{DISCUSSION}

With the aid of the stationary phase method, the single trace response of the phase-shift migration (equation 1 ) becomes equation 5 , which can be used directly for the Kirchhoff prestack time migration. The amplitude term in equation 5 corresponds to the geometric spreading of seismic rays. The phase term in this equation is dependent on the diffraction traveltime if we do not consider the influence of $\xi$. The amplitude and traveltime of the migration operator are expressed in terms of the horizontal slowness components of source and receiver. In this paper, we mainly present an analytic formula for the diffraction traveltime of $\mathrm{P}$-waves in orthorhombic media in terms of midpoint and source-receiver 
half-offset. In this process, the horizontal slowness components of source and receiver are analytically linked to the midpoint and source-receiver half-offset using a perturbation theory and the Shanks transform. The relevant derivations are shown in the "Slowness approximation at stationary point" section. The slowness

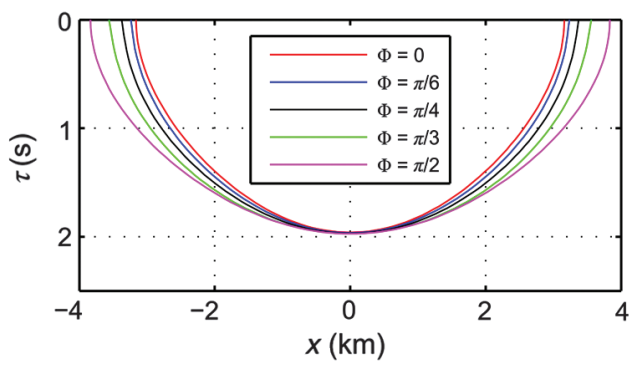

Figure 5. The migration isochrones along different acquisition azimuths in a homogeneous orthorhombic media. The source-receiver offset is taken as $0.5 \mathrm{~km}$. The lateral axis $x$ is the spatial position. The vertical axis $\tau$ is the two-way vertical traveltime describing the time depth of a point diffractor. The medium parameters are the same as in Figure 3.
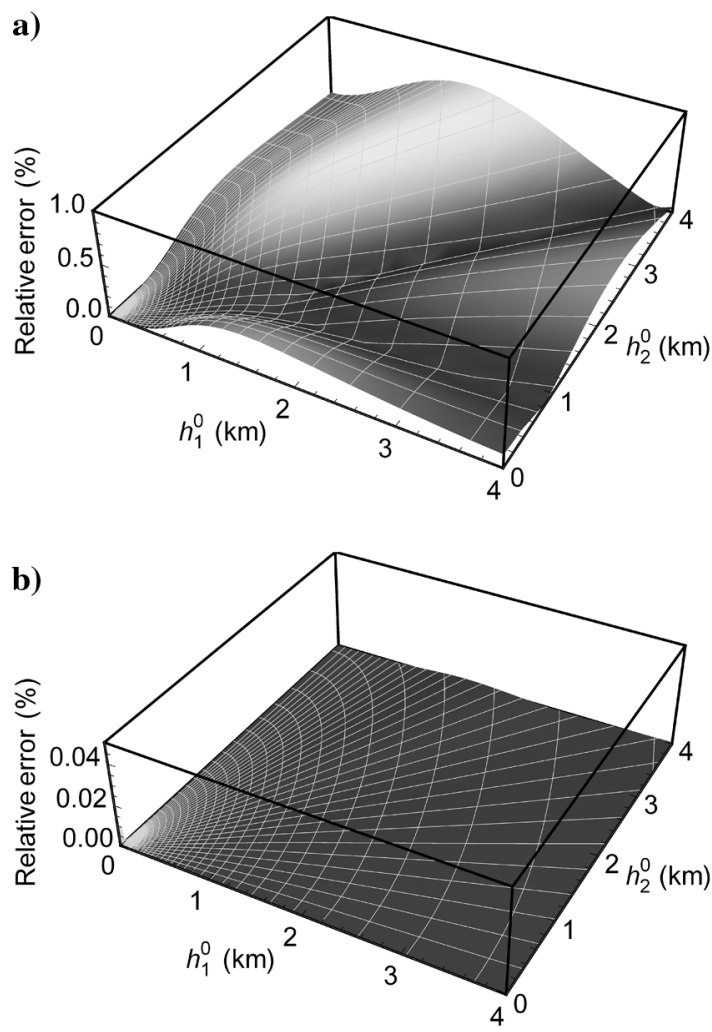

Figure 6. The relative error comparison between (a) the nonhyperbolic moveout approximation (Xu et al., 2005; Vasconcelos and Tsvankin, 2006) and (b) approximation 17 for the P-wave traveltime in a horizontal reflector in a $3 \mathrm{D}$ orthorhombic medium. The lateral coordinates $h_{1}^{0}$ and $h_{2}^{0}$ denote the projections of source-receiver half-offset on $x$ - and $z$-axes. The model parameters include $v_{p 0}=3 \mathrm{~km} / \mathrm{s}, v_{n 1}=3.5 \mathrm{~km} / \mathrm{s}, v_{n 2}=2.5 \mathrm{~km} / \mathrm{s}, \eta_{1}=0.1$, $\eta_{2}=0.1$, and $\eta_{3}=0.1$. The principal axes of orthorhombic model coincide with the acquisition axes. The zero-offset two-way traveltime is $\tau=0.667 \mathrm{~s}$.
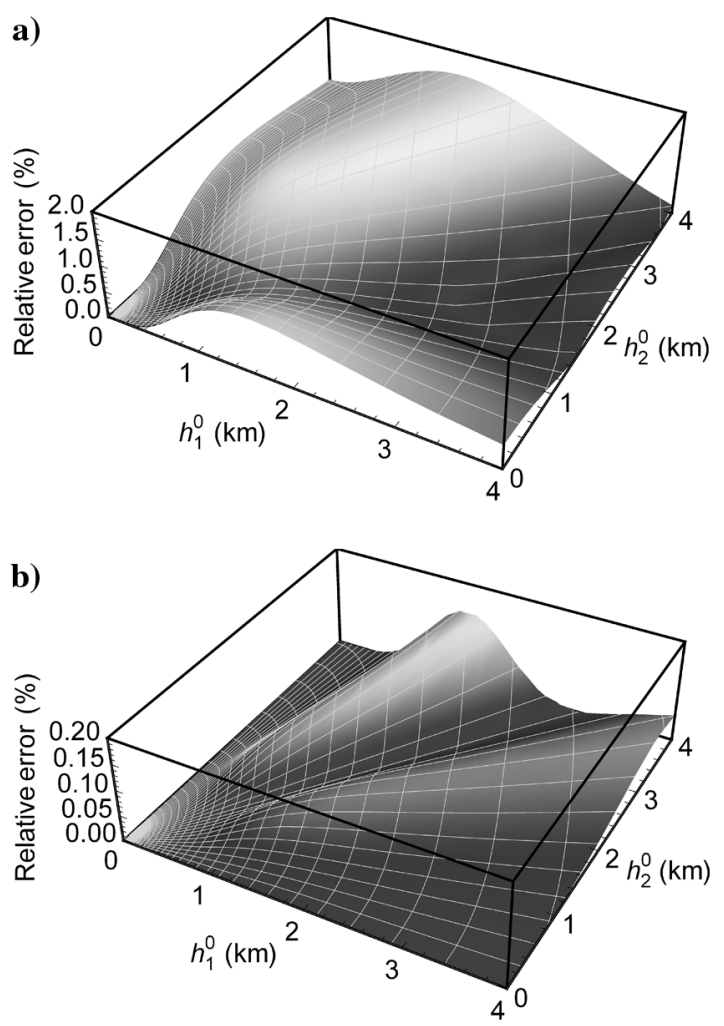

Figure 7. Similar to Figure 6, but the anellipticity parameters of orthorhombic model are $\eta_{1}=0.2, \eta_{2}=0.2$, and $\eta_{3}=0.2$.
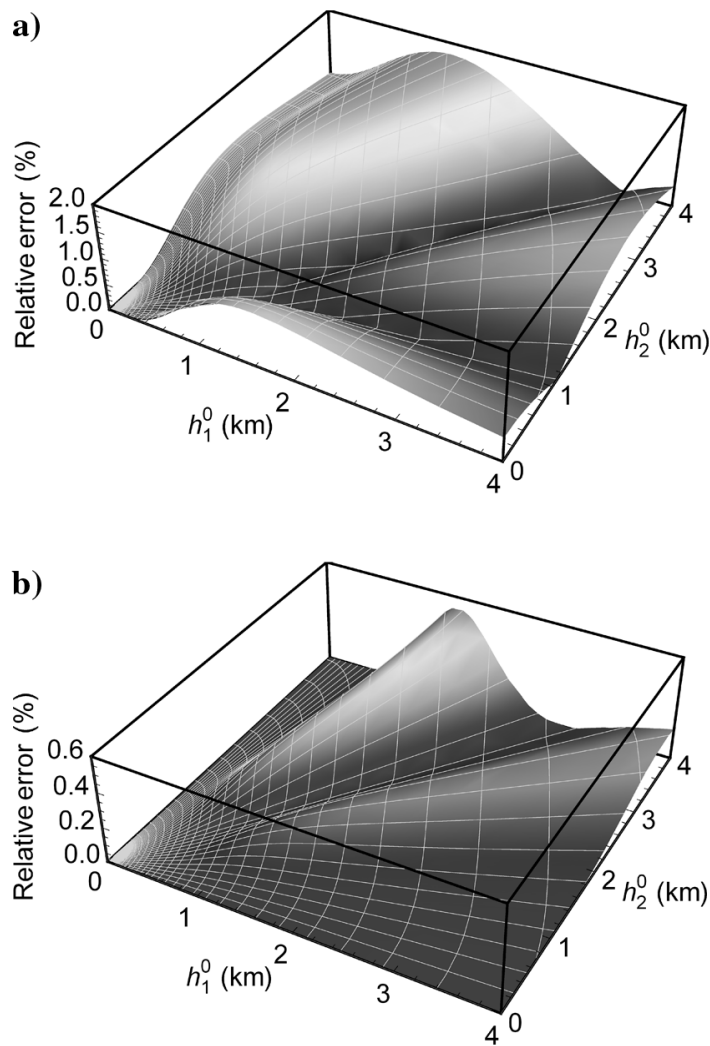

Figure 8. Similar to Figure 6, but the anellipticity parameters of orthorhombic model are $\eta_{1}=0.2, \eta_{2}=0.2$, and $\eta_{3}=0.3$. 
approximation presented in this section is also helpful for determining the amplitude term in equation 5 because Gaussian curvatures $\lambda_{s}$ and $\lambda_{g}$ are related to the second-order derivatives of vertical slowness with respect to the horizontal slowness (see equation A-13 with equations A-5 and 6-9). This means that for the given midpoint and source-receiver half-offset, calculating amplitude can be achieved in an entirely analytic and straightforward way similar to calculating traveltime, although it is relatively complex compared with the traveltime. Meanwhile, we note that for vertical orthorhombic media, the vertical velocity parameter $v_{p 0}$ can be eliminated in the expressions for amplitude and traveltime. This indicates that Kirchhoff prestack time migration of $\mathrm{P}$-waves in vertical orthorhombic media requires only seven independent parameters (including two-way zero-offset traveltime $\tau$, NMO velocities $v_{n 1}$ and $v_{n 2}$, three anellipticity parameters $\eta_{1}, \eta_{2}, \eta_{3}$, and the azimuth $\phi$ of an orthorhombic medium). For homogeneous orthorhombic media, these parameters except $\tau$ are the real medium parameters. For heterogeneous orthorhombic media, all these parameters are effective parameters that are inverted by the moveout velocity analysis (Grechka and Tsvankin, 1999; Vasconcelos and Tsvankin, 2006; Yan and Tsvankin, 2008). An example of Kirchhoff prestack time-domain migration for VTI media is shown in Alkhalifah (2000b).

"Numerical examples" section shows that the anellipticity of orthorhombic media strongly affects the accuracy of the offset-midpoint traveltime approximation when the source-receiver half-offset and the lateral distance between the midpoint and the point diffractor are very large: the stronger the anellipticity, the less accurate the traveltime approximation. To purse a more accurate traveltime, we
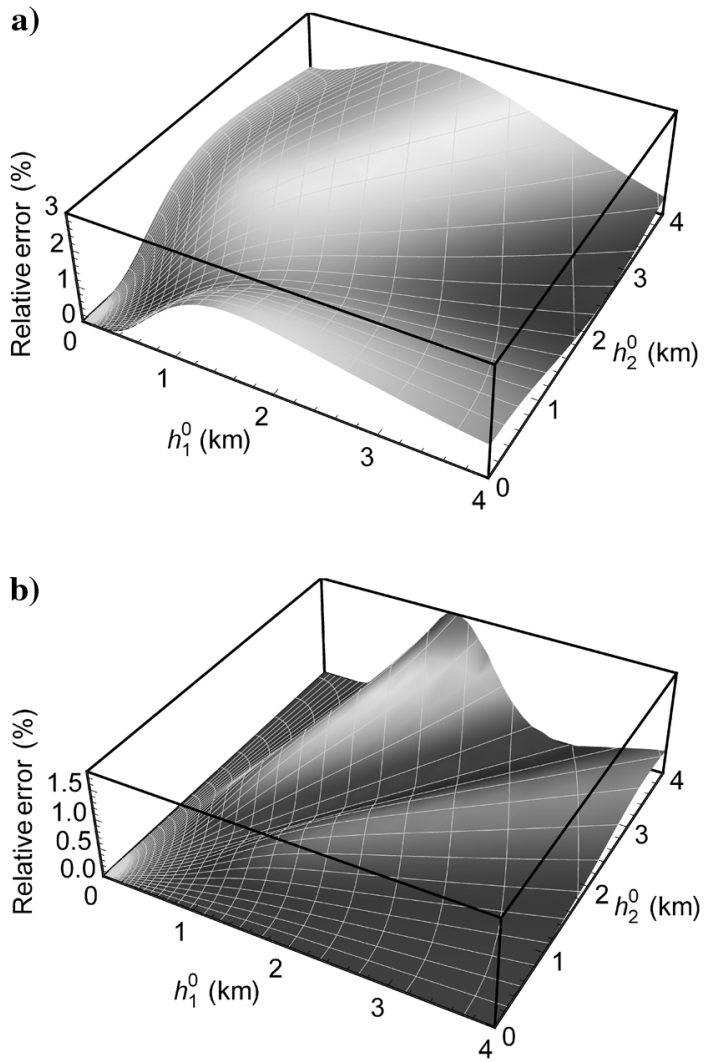

Figure 9. Similar to Figure 6 , but the anellipticity parameters of orthorhombic model are $\eta_{1}=0.3, \eta_{2}=0.3$, and $\eta_{3}=0.3$. may continue to determine the third-order expansions of the horizontal slowness components squared with respect to the anellipticity parameters, which is a direct extension of the method introduced in Appendix A. We may use the Shanks transform to improve the accuracy of the third-order expansions. In this case, application of the Shanks transform can also be seen in (Bender and Orszag [1978], pp. 369-375). Once the horizontal slowness components are obtained, we may use equation 17 to calculate the traveltime. As an alternative, we may also numerically calculate the horizontal slowness components for source and receiver. In Appendix C, equations $\mathrm{C}-1$ and $\mathrm{C}-2$ are two nonlinear algebraic equations with respect to the horizontal slowness squared. We may adopt iterative numerical methods, such as Gaussian-Newton method and conjugate gradient method to solve the equations C-1 and C-2. The analytic and exact horizontal slowness components for an elliptically background, which are obtained by setting $\eta_{1}=\eta_{2}=\eta_{3}=0$ in equations C-7 and C-8, may be used as the initial values of the horizontal slowness components for the iterative numerical methods.

The formulas for amplitude and traveltime derived in this paper can also be used in the angle-domain prestack time migration. Compared with the conventional time migration discussed above, the angle-domain prestack time migration requires the offset-to-angle mapping of seismic image. In this process, the local scattering angle and azimuth are calculated from the slowness vectors of source and receiver (Cheng et al., 2011, 2012; Sun and Sun 2015). In this paper, we show that the horizontal slowness components of source and receiver can be analytically expressed in terms of the coordinates of midpoint and source-receiver half-offset. From slowness surface equation 6 with equations 7-9, the vertical slowness component can be further obtained from the horizontal slowness components. Therefore, the offset-to-angle mapping of the seismic image can be eventually achieved. The last example in the "Numerical examples" section illustrates the accuracy of approximate formulas 15 and 16 for horizontal slowness components in vertical orthorhombic media with weakly or moderate anisotropy. This guarantees the accuracy of offset-to-angle mapping if we do not consider the error in the model parameters. Similar to Kirchhoff prestack time migration discussed in the previous paragraph, the vertical velocity parameter $v_{p 0}$ is not required for angle-domain Kirchhoff prestack time migration.

\section{CONCLUSION}

With the aid of a perturbation theory and the Shanks transform, we derive an analytic offset-midpoint traveltime formula from the single trace response of the phase-shift midpoint-offset migration using the stationary phase approximation. The formula is valid for homogeneous orthorhombic media with weak to moderate anellipticity. Also, the formula may be used in the prestack time-domain Kirchhoff migration in heterogeneous orthorhombic media, in which the medium parameters are replaced by their effective parameters obtained from the semblance-based velocity analysis.

\section{ACKNOWLEDGMENTS}

Q. Hao and A. Stovas thank the Rock Seismic Research Project for financial support. T. Alkhalifah thanks KAUST for its support. We are very grateful to an anonymous associate editor, three anonymous reviewers, and Y. Sripanich for many valuable suggestions. 


\section{APPENDIX A}

\section{KIRCHHOFF'S PRESTACK TIME MIGRATION}

In this section, we derive a closed-form expression for the singletrace response of the prestack time-domain phase-shift migration using the stationary phase method. We adopt the special Fourier transform convention used in Yilmaz and Claerbout (1980), Alkhalifah (2000a), Yilmaz (2001, p. 156), and Hao et al. (2015) neglecting the factor $1 /(2 \pi)$ in front of the inverse of Fourier transform for convenience. The stationary phase method for oscillatory integrals is discussed by Bleistein and Handelsman (1986) in detail.

We change the offset-midpoint domain phase-shift migration 1 of a single trace back to the source-receiver domain (Hao et al., 2015),

$$
\begin{aligned}
& P\left(x_{1}, x_{2}, h_{1}=0, h_{2}=0, \tau, t=0\right) \\
& \quad=\int_{-\infty}^{\infty} \int_{-\infty}^{\infty} \int_{-\infty}^{\infty} \int_{-\infty}^{\infty} \int_{-\infty}^{\infty} \tilde{P}\left(x_{1}^{0}, x_{2}^{0}, h_{1}^{0}, h_{2}^{0}, \tau=0, \omega\right) \\
& \quad \times \exp (i \omega T) d \omega d k_{s 1} d k_{s 2} d k_{g 1} d k_{g 2},
\end{aligned}
$$

where $\left(k_{s 1}, k_{s 2}\right)=\omega\left(p_{s 1}, p_{s 2}\right)$ and $\left(k_{g 1}, k_{g 2}\right)=\omega\left(p_{g 1}, p_{g 2}\right)$ are the vectors of horizontal wavenumbers of source and receiver. $\left(p_{s 1}, p_{s 2}\right)$ and $\left(p_{g 1}, p_{g 2}\right)$ are the corresponding horizontal slowness vectors and $T$ is the plane wave traveltime given by equation 2 .

The stationary phase method requires the first-order derivative of $\omega T$ with respect to the horizontal wavenumbers of source and receiver equal to zero. From the traveltime expression 2, we find the following stationary phase condition:

$$
\frac{\partial q_{(s, g)}}{\partial p_{i(s, g)}}=-\frac{2 y_{i(s, g)}}{\tau v_{p 0}}, \quad i=1,2
$$

where subscripts $s$ and $g$ correspond to source and receiver, respectively, the expressions for $y_{i(s, g)}$ are given after equation 2 . The $\mathrm{P}$ wave slownesses for the source and receiver at the stationary point $\left(\tilde{p}_{1(s, g)}, \tilde{p}_{2(s, g)}, \tilde{q}_{(s, g)}\right)$ can be uniquely determined from equation A-2 and the slowness surface equation. For orthorhombic media, the slowness surface equation is given by equations $6-9$.

We expand the plane-wave traveltime function $T$ in equation A-1 to second order at the stationary point

$$
\begin{aligned}
T \approx & \tilde{T}+\frac{1}{2}\left(\boldsymbol{p}_{s}-\tilde{\boldsymbol{p}}_{s}\right) \boldsymbol{T}_{s s}\left(\boldsymbol{p}_{s}-\tilde{\boldsymbol{p}}_{s}\right)^{T} \\
& +\frac{1}{2}\left(\boldsymbol{p}_{g}-\tilde{\boldsymbol{p}}_{g}\right) \boldsymbol{T}_{g g}\left(\boldsymbol{p}_{g}-\tilde{\boldsymbol{p}}_{g}\right)^{T}
\end{aligned}
$$

where $\boldsymbol{p}_{s}=\left(p_{s 1}, p_{s 2}\right)$ and $\boldsymbol{p}_{g}=\left(p_{g 1}, p_{g 2}\right)$ denote the horizontal slowness vectors of plane waves for source and receiver, respectively; $\tilde{\boldsymbol{p}}_{s}=\left(\tilde{p}_{s 1}, \tilde{p}_{s 2}\right)$ and $\tilde{\boldsymbol{p}}_{g}=\left(\tilde{p}_{g 1}, \tilde{p}_{g 2}\right)$ denote the horizontal slowness vectors for source and receiver, respectively, satisfying the stationary phase condition A-2; $\tilde{T}=T\left(\tilde{p}_{s 1}, \tilde{p}_{s 2}, \tilde{p}_{g 1}, \tilde{p}_{g 2}\right)$ denotes the traveltime at stationary point, which is identical to the diffraction traveltime of seismic rays; and $\boldsymbol{T}_{s s}$ and $\boldsymbol{T}_{g g}$ are symmetric matrices of second-order traveltime derivatives,

$$
\begin{gathered}
\boldsymbol{T}_{s s}=\left.\left(\begin{array}{cc}
\frac{\partial^{2} T}{\partial p_{s 1}^{2}} & \frac{\partial^{2} T}{\partial p_{s 1} \partial p_{s 2}} \\
\frac{\partial^{2} T}{\partial p_{s 1} \partial p_{s 2}} & \frac{\partial^{2} T}{\partial p_{s 2}^{2}}
\end{array}\right)\right|_{p_{s 1}=\tilde{p}_{s 1}, p_{s 2}=\tilde{p}_{s 2}}, \\
\boldsymbol{T}_{g g}=\left.\left(\begin{array}{cc}
\frac{\partial^{2} T}{\partial p_{g 1}^{2}} & \frac{\partial^{2} T}{\partial p_{g 1} \partial p_{g 2}} \\
\frac{\partial^{2} T}{\partial p_{g 1} \partial p_{g 2}} & \frac{\partial^{2} T}{\partial p_{g 2}^{2}}
\end{array}\right)\right|_{p_{g 1}=\tilde{p}_{g 1}, p_{g 2}=\tilde{p}_{g 2}}
\end{gathered} .
$$

Substituting the traveltime equation 2, the elements in matrices in equation A-4 are expressed in terms of the horizontal slowness components of the source and receiver,

$$
\frac{\partial^{2} T}{\partial p_{i(s, g)} \partial p_{j(s, g)}}=\frac{\tau v_{p 0}}{2} \frac{\partial^{2} q_{(s, g)}}{\partial p_{i(s, g)} \partial p_{j(s, g)}}, \quad i, j=1,2 .
$$

We consider the eigen decomposition of symmetric matrices $\boldsymbol{T}_{s s}$ and $\boldsymbol{T}_{g g}$,

$$
\boldsymbol{T}_{s s}=\boldsymbol{Q}_{s} \lambda_{s} \boldsymbol{Q}_{s}^{T}, \boldsymbol{T}_{g g}=\boldsymbol{Q}_{g} \lambda_{g} \boldsymbol{Q}_{g}^{T}
$$

where $\boldsymbol{Q}_{s}$ and $\boldsymbol{Q}_{g}$ are the orthogonal matrices composed of the eigenvectors of $\boldsymbol{T}_{s s}$ and $\boldsymbol{T}_{g g} ; \lambda_{s}$ and $\lambda_{g}$ are the diagonal matrices composed of the eigenvalues of $\boldsymbol{T}_{s s}$ and $\boldsymbol{T}_{g g}$,

$$
\lambda_{s}=\left(\begin{array}{ll}
\lambda_{s 1} & \\
& \lambda_{s 2}
\end{array}\right), \quad \lambda_{g}=\left(\begin{array}{ll}
\lambda_{g 1} & \\
& \lambda_{g 2}
\end{array}\right),
$$

where matrices $\lambda_{s}$ and $\lambda_{g}$ are real valued and linked to the principal curvatures of the slowness surfaces for the source and receiver.

Let us introduce two vectors $\boldsymbol{\mu}_{s}=\left(\mu_{s 1}, \mu_{s 2}\right), \boldsymbol{\mu}_{g}=\left(\mu_{g 1}, \mu_{g 2}\right)$ defined by

$$
\boldsymbol{\mu}_{s}^{T}=\boldsymbol{Q}_{s}^{T}\left(\boldsymbol{p}_{s}-\tilde{\boldsymbol{p}}_{s}\right)^{T}, \quad \boldsymbol{\mu}_{g}^{T}=\boldsymbol{Q}_{g}^{T}\left(\boldsymbol{p}_{g}-\tilde{\boldsymbol{p}}_{g}\right)^{T},
$$

such that traveltime equation A-3 can be rewritten as

$$
T \approx \tilde{T}+\frac{1}{2} \lambda_{s 1} \mu_{s 1}^{2}+\frac{1}{2} \lambda_{s 2} \mu_{s 2}^{2}+\frac{1}{2} \lambda_{g 1} \mu_{g 1}^{2}+\frac{1}{2} \lambda_{g 2} \mu_{g 2}^{2},
$$

and the integration variables in A-1 can be changed

$$
\begin{aligned}
P( & \left.x_{1}, x_{2}, h_{1}=0, h_{2}=0, \tau, t=0\right) \\
\quad & =\int_{-\infty}^{\infty} \int_{-\infty}^{\infty} \int_{-\infty}^{\infty} \int_{-\infty}^{\infty} \int_{-\infty}^{\infty} \omega^{4} \tilde{P}\left(x_{1}^{0}, x_{2}^{0}, h_{1}^{0}, h_{2}^{0}, \tau=0, \omega\right) \\
& \times \exp (i \omega T) d \omega d \mu_{s 1} d \mu_{s 2} d \mu_{g 1} d \mu_{g 2} .
\end{aligned}
$$

Considering the fact that

$$
\int_{-\infty}^{\infty} \exp \left(i \lambda u^{2}\right) d u=\sqrt{\pi /|\lambda|} \exp \left(\frac{1}{4} \pi i \operatorname{sgn} \lambda\right),
$$

and equation A-9, equation A-10 is approximated by

$$
\begin{aligned}
& P\left(x_{1}, x_{2}, h_{1}=0, h_{2}=0, \tau, t=0\right) \\
& \quad \approx \frac{1}{4 \pi^{2} \sqrt{\left|\lambda_{s} \lambda_{g}\right|}} \frac{\partial^{2}}{\partial \tilde{T}^{2}} \tilde{P}\left(x_{1}^{0}, x_{2}^{0}, h_{1}^{0}, h_{2}^{0}, \tau=0, \tilde{T}\right) \cos \xi
\end{aligned}
$$


where $\lambda_{s}$ and $\lambda_{g}$ are linked to the Gaussian curvatures of the slowness surfaces for the source and receiver

$$
\begin{aligned}
& \lambda_{s}=\lambda_{s 1} \lambda_{s 2}=\left.\left(\frac{\partial^{2} T}{\partial p_{s 1}^{2}} \frac{\partial^{2} T}{\partial p_{s 2}^{2}}-\left(\frac{\partial^{2} T}{\partial p_{s 1} \partial p_{s 2}}\right)^{2}\right)\right|_{p_{s 1}=\tilde{p}_{s 1}, p_{s 2}=\tilde{p}_{s 2}}, \\
& \lambda_{g}=\lambda_{g 1} \lambda_{g 2}=\left.\left(\frac{\partial^{2} T}{\partial p_{g 1}^{2}} \frac{\partial^{2} T}{\partial p_{g 2}^{2}}-\left(\frac{\partial^{2} T}{\partial p_{g 1} \partial p_{g 2}}\right)^{2}\right)\right|_{p_{g 1}=\tilde{p}_{g 1}, p_{g 2}=\tilde{p}_{g 2}},
\end{aligned}
$$

and the phase shift $\xi$ is given by

$$
\xi=\pi+\frac{\pi}{4}\left(\operatorname{sgn}\left(\lambda_{s 1}\right)+\operatorname{sgn}\left(\lambda_{s 2}\right)+\operatorname{sgn}\left(\lambda_{g 1}\right)+\operatorname{sgn}\left(\lambda_{g 2}\right)\right) .
$$

Formula A-12 describes the single-trace response of Kirchhoff prestack time migration for general homogeneous anisotropic media through mapping the time-domain seismic data at surface $(\tau=0)$ to subsurface. For a given pair of midpoint and sourcereceiver offset, the Gaussian curvatures $\lambda_{s}$ and $\lambda_{g}$, and traveltime $\tilde{T}$ need the source and receiver slownesses that are preliminarily estimated from the stationary phase condition A-2 and the slowness surface equation for the considered anisotropic media. The process of estimating source and receiver slownesses is generally not straightforward. The main reason is that there is no exact and explicit expression for phase propagation direction in terms of the group propagation direction in general anisotropic media.

\section{APPENDIX B}

\section{THE MODIFIED ALKHALIFAH'S NOTATION FOR ACOUSTIC ORTHORHOMBIC MEDIA}

The Alkhalifah's (2003) notation is used to describe an acoustic orthorhombic medium. For this set of parameters, the S-wave velocity along its principal axes is assumed to be zero. Therefore, only six independent parameters are required to describe $\mathrm{P}$-wave kinematics. We slightly modify the Alkhalifah's notation for acoustic orthorhombic media:

$$
\begin{gathered}
v_{p 0} \equiv \sqrt{a_{33}}, \\
\eta_{1} \equiv \frac{\varepsilon_{1}-\delta_{1}}{1+2 \delta_{1}}=\frac{a_{22}\left(a_{33}-a_{44}\right)}{2\left(a_{23}^{2}+2 a_{23} a_{44}+a_{33} a_{44}\right)}-\frac{1}{2}, \\
\eta_{2} \equiv \frac{\varepsilon_{2}-\delta_{2}}{1+2 \delta_{2}}=\frac{a_{11}\left(a_{33}-a_{55}\right)}{2\left(a_{13}^{2}+2 a_{13} a_{55}+a_{33} a_{55}\right)}-\frac{1}{2}, \\
\eta_{3} \equiv \frac{\varepsilon_{1}-\varepsilon_{2}-\delta_{3}\left(1+2 \varepsilon_{2}\right)}{\left(1+2 \delta_{3}\right)\left(1+2 \delta_{2}\right)}=\frac{a_{22}\left(a_{11}-a_{66}\right)}{2\left(a_{12}^{2}+2 a_{12} a_{66}+a_{11} a_{66}\right)}-\frac{1}{2},
\end{gathered}
$$

$$
\begin{aligned}
& v_{n 1} \equiv v_{p 0} \sqrt{1+2 \delta_{1}}=\sqrt{\frac{a_{23}^{2}+2 a_{23} a_{44}+a_{33} a_{44}}{a_{33}-a_{44}}}, \\
& v_{n 2} \equiv v_{p 0} \sqrt{1+2 \delta_{2}}=\sqrt{\frac{a_{13}^{2}+2 a_{13} a_{55}+a_{33} a_{55}}{a_{33}-a_{55}}},
\end{aligned}
$$

where $a_{i j}$ denotes the density-normalized stiffness coefficients in Voigt notation; $v_{p 0}$ denotes the phase velocity of $\mathrm{P}$-waves along VTI (z-axis); subscripts 1, 2, 3 except for $a_{i j}$ correspond to the $[y, z],[x, z]$, and $[x, y]$ symmetry planes of an orthorhombic medium, respectively; $\varepsilon_{i}(i=1,2 i)$ and $\delta_{i}(i=1,2,3)$ are the Thomsen-type parameters for orthorhombic media (Tsvankin, 1997); $\eta_{i}(i=1,2,3)$ denote the anellipticity parameters (Grechka and Tsvankin, 1999); and $v_{n i}(i=1,2)$ denote the NMO velocities.

\section{APPENDIX C}

\section{THE PERTURBATION EXPANSIONS OF HORIZONTAL SLOWNESS SQUARED}

In this appendix, we derive the perturbation expansions of the horizontal slowness squared defined in equations 10 and 11 .

Substituting equation 6 with 7 and 8 into equation 4 allows us to derive two algebraic equations:

$$
\begin{aligned}
& a\left(1+2 \eta_{3}\right) A B^{3}-p_{1}^{2} v_{n 2}^{4} C^{2}=0, \\
& b\left(1+2 \eta_{3}\right) A B^{3}-p_{2}^{2} v_{n 1}^{4} D^{2}=0,
\end{aligned}
$$

where $a=\left(2 y_{1} / \tau\right)^{2}$ and $b=\left(2 y_{2} / \tau\right)^{2}$; and

$$
\begin{aligned}
A= & \left(1+2 \eta_{3}\right)\left(1-v_{n 2}^{2} p_{1}^{2}\left(1+2 \eta_{2}\right)-v_{n 1}^{2} p_{2}^{2}\left(1+2 \eta_{1}\right)\right) \\
& +2 v_{n 1}^{2} v_{n 2}^{2} p_{1}^{2} p_{2}^{2} \eta_{3}\left(1+2 \eta_{1}\right)\left(1+2 \eta_{2}\right) \\
& B=1-2 v_{n 2}^{2} p_{1}^{2} \eta_{2}-2 v_{n 1}^{2} p_{2}^{2} \eta_{1}-\Omega v_{n 1}^{2} v_{n 2}^{2} p_{1}^{2} p_{2}^{2}
\end{aligned}
$$

$$
\begin{aligned}
C= & 1+2 \eta_{3}+p_{2}^{4} v_{n 1}^{4}\left(1+2 \eta_{1}\right)\left(4 \eta_{1} \eta_{3}\left(1+2 \eta_{2}\right)+\Omega\left(1+2 \eta_{3}\right)\right) \\
& -p_{2}^{2} v_{n 1}^{2}\left(2\left(\eta_{1}-\eta_{2}\right)+2 \eta_{3}\left(1+4 \eta_{1}+4 \eta_{1} \eta_{2}\right)+\Omega\left(1+2 \eta_{3}\right)\right),
\end{aligned}
$$

$$
\begin{aligned}
D= & 1+2 \eta_{3}+p_{1}^{4} v_{n 2}^{4}\left(1+2 \eta_{2}\right)\left(4 \eta_{2} \eta_{3}\left(1+2 \eta_{1}\right)+\Omega\left(1+2 \eta_{3}\right)\right) \\
& -p_{1}^{2} v_{n 2}^{2}\left(2\left(\eta_{2}-\eta_{1}\right)+2 \eta_{3}\left(1+4 \eta_{2}+4 \eta_{1} \eta_{2}\right)+\Omega\left(1+2 \eta_{3}\right)\right) .
\end{aligned}
$$

In equations C-4-C-6, the expression for $\Omega$ is given in equation 9 . Equations $\mathrm{C}-1$ and $\mathrm{C}-2$ are two eighth-order algebraic equations in terms of $p_{1}^{2}$ and $p_{2}^{2}$. It is difficult to obtain analytic and simple solutions for these equations. We use perturbation theory to seek approximate solutions of equations $\mathrm{C}-1$ and $\mathrm{C}-2$. As shown in equations 10 and 11, we define the perturbation expansions of $p_{1}^{2}$ and $p_{2}^{2}$ with respect to anellipticity parameters $\eta_{i}, i=1,2,3$, 


$$
\begin{aligned}
& p_{1}^{2}=c_{0}+\sum_{i=1}^{3} c_{i} \eta_{i}+\sum_{i, j=1, i \leq j}^{3} c_{i j} \eta_{i} \eta_{j} \\
& p_{2}^{2}=d_{0}+\sum_{i=1}^{3} d_{i} \eta_{i}+\sum_{i, j=1, i \leq j}^{3} d_{i j} \eta_{i} \eta_{j}
\end{aligned}
$$

where $c_{0}, c_{i}, c_{i j}, d_{0}, d_{i}$, and $d_{i j}$ are the undetermined perturbation coefficients. Substituting perturbations C-7 and C-8 into equations $\mathrm{C}-1$ and $\mathrm{C}-2$, we can obtain two new second-order expansions with respect to the three anellipticity parameters $\eta_{i}$. Because all coefficients in the two expansions are equal to zero, we can determine the coefficients defined in equations C-7 and C-8. The explicit expressions for these coefficients are shown as follows.

The expressions for the coefficients in equation C-7 are given by The zero-order coefficient:

$$
c_{0}=v_{n 2}^{-2} \kappa^{-1} a v_{n 1}^{2} .
$$

The first-order coefficients:

$$
\begin{gathered}
c_{1}=2 \kappa^{-3} a b v_{n 1}^{2}\left(a v_{n 1}^{2}+b v_{n 2}^{2}-2 v_{n 1}^{2} v_{n 2}^{2}\right), \\
c_{2}=-2 \kappa^{-3} v_{n 2}^{-2} a v_{n 1}^{2}\left(a^{2} v_{n 1}^{4}+a v_{n 1}^{2}\left(3 b+4 v_{n 1}^{2}\right) v_{n 2}^{2}\right. \\
\left.+2 b\left(b+v_{n 1}^{2}\right) v_{n 2}^{4}\right), \\
c_{3}=-2 \kappa^{-3} a b v_{n 1}^{2}\left(a v_{n 1}^{2}-2\left(b+v_{n 1}^{2}\right) v_{n 2}^{2}\right) .
\end{gathered}
$$

The second-order coefficients:

$$
\begin{array}{rl}
c_{11}= & \kappa^{-5} a b v_{n 1}^{4}\left(-4 a^{3} v_{n 1}^{4}+a^{2} v_{n 1}^{2} v_{n 2}^{2}\left(-8 b+9 v_{n 1}^{2}\right)\right. \\
+ & a v_{n 2}^{4}\left(-4 b^{2}-25 b v_{n 1}^{2}+15 v_{n 1}^{4}\right) \\
+ & \left.2 v_{n 2}^{6}\left(-17 b^{2}+20 b v_{n 1}^{2}+v_{n 1}^{4}\right)\right) \\
c_{22}= & \kappa^{-5} v_{n 2}^{-2} a v_{n 1}^{2}\left(4 a^{4} v_{n 1}^{8}+20 a^{3} v_{n 1}^{6} v_{n 2}^{2}\left(b+v_{n 1}^{2}\right)\right. \\
+ & a^{2} v_{n 1}^{4} v_{n 2}^{4}\left(40 b^{2}+109 b v_{n 1}^{2}+88 v_{n 1}^{4}\right) \\
+ & a b v_{n 1}^{2} v_{n 2}^{6}\left(b+v_{n 1}^{2}\right)\left(36 b+79 v_{n 1}^{2}\right) \\
+ & \left.2 b v_{n 2}^{8}\left(b+v_{n 1}^{2}\right)^{2}\left(6 b+v_{n 1}^{2}\right)\right) \\
c_{33}= & \kappa^{-5} a b v_{n 1}^{2} v_{n 2}^{2}\left(a^{2} v_{n 1}^{4}\left(28 b+9 v_{n 1}^{2}\right)\right. \\
& +a v_{n 1}^{2} v_{n 2}^{2}\left(b+v_{n 1}^{2}\right)\left(-40 b+3 v_{n 1}^{2}\right) \\
& \left.+2 v_{n 2}^{4}\left(2 b-3 v_{n 1}^{2}\right)\left(b+v_{n 1}^{2}\right)^{2}\right) \\
c_{12}= & -2 \kappa^{-5} a b v_{n 1}^{2} v_{n 2}^{2}\left(a^{2} v_{n 1}^{4}\left(4 b+29 v_{n 1}^{2}\right)\right. \\
& +a v_{n 1}^{2} v_{n 2}^{2}\left(8 b^{2}+15 b v_{n 1}^{2}-41 v_{n 1}^{2}\right) \\
& \left.+2 v_{n 2}^{4}\left(b+v_{n 1}^{2}\right)\left(2 b^{2}-9 b v_{n 1}^{2}+v_{n 1}^{4}\right)\right) \\
c_{13}=2 \kappa^{-5} & a b v_{n 1}^{2}\left(2 a^{3} v_{n 1}^{6}-a^{2} v_{n 1}^{4} v_{n 2}^{2}\left(10 b+9 v_{n 1}^{2}\right)\right. \\
+ & a v_{n 1}^{2} v_{n 2}^{4}\left(-8 b^{2}+31 b v_{n 1}^{2}-9 v_{n 1}^{4}\right) \\
\left.+2 v_{n 2}^{6}\left(b+v_{n 1}^{2}\right)\left(2 b^{2}-9 b v_{n 1}^{2}+v_{n 1}^{4}\right)\right) \\
& \\
& \\
&
\end{array}
$$

$$
\begin{aligned}
c_{23}= & 2 \kappa^{-5} a b v_{n 1}^{2} v_{n 2}^{2}\left(a^{2} v_{n 1}^{4}\left(10 b+29 v_{n 1}^{2}\right)\right. \\
& +a v_{n 1}^{2} v_{n 2}^{2}\left(2 b-41 v_{n 1}^{2}\right)\left(b+v_{n 1}^{2}\right) \\
& \left.+2 v_{n 2}^{4}\left(-4 b+v_{n 1}^{2}\right)\left(b+v_{n 1}^{2}\right)^{2}\right) .
\end{aligned}
$$

In equations C-9-C-18, the quantity $\kappa$ is given by

$$
\kappa=a v_{n 1}^{2}+b v_{n 2}^{2}+v_{n 1}^{2} v_{n 2}^{2} .
$$

The expressions for the coefficients in expansion C-8 are given by the zero-order coefficient:

$$
d_{0}=v_{n 1}^{-2} \kappa^{-1} b v_{n 2}^{2}
$$

The first-order coefficients:

$$
\begin{gathered}
d_{1}=-2 \kappa^{-3} v_{n 1}^{-2} b v_{n 2}^{2}\left(2 a^{2} v_{n 1}^{4}+a v_{n 1}^{2} v_{n 2}^{2}\left(3 b+2 v_{n 1}^{2}\right)\right. \\
\left.+b v_{n 2}^{4}\left(b+4 v_{n 1}^{2}\right)\right), \\
d_{2}=2 \kappa^{-3} a b v_{n 2}^{2}\left(a v_{n 1}^{2}+v_{n 2}^{2}\left(b-2 v_{n 1}^{2}\right)\right), \\
d_{3}=2 \kappa^{-3} a b v_{n 2}^{2}\left(-b v_{n 2}^{2}+2 v_{n 1}^{2}\left(a+v_{n 2}^{2}\right)\right) .
\end{gathered}
$$

The second-order coefficients:

$$
\begin{aligned}
d_{11}= & \kappa^{-5} v_{n 1}^{-2} b v_{n 2}^{2}\left(12 a^{4} v_{n 1}^{8}+2 a^{3} v_{n 1}^{6} v_{n 2}^{2}\left(18 b+13 v_{n 1}^{2}\right)\right. \\
& +a^{2} v_{n 1}^{4} v_{n 2}^{4}\left(40 b^{2}+115 b v_{n 1}^{2}+16 v_{n 1}^{4}\right) \\
& +a v_{n 1}^{2} v_{n 2}^{6}\left(20 b^{3}+109 b^{2} v_{n 1}^{2}+79 b v_{n 1}^{4}+2 v_{n 1}^{6}\right) \\
& \left.+4 b^{2} v_{n 2}^{8}\left(b^{2}+5 b v_{n 1}^{2}+22 v_{n 1}^{4}\right)\right) \\
d_{22}= & \kappa^{-5} a b v_{n 2}^{4}\left(-2 a^{2} v_{n 1}^{4}\left(2 b+17 v_{n 1}^{2}\right)\right. \\
& +a v_{n 1}^{2} v_{n 2}^{2}\left(-8 b^{2}-25 b v_{n 1}^{2}+40 v_{n 1}^{4}\right) \\
& \left.+v_{n 2}^{4}\left(b+v_{n 1}^{2}\right)\left(-4 b^{2}+13 b v_{n 1}^{2}+2 v_{n 1}^{4}\right)\right)
\end{aligned}
$$

$$
\begin{aligned}
d_{33}= & \kappa^{-5} a b v_{n 1}^{2} v_{n 2}^{2}\left(4 a^{3} v_{n 1}^{4}+2 a^{2} v_{n 1}^{2} v_{n 2}^{2}\left(-20 b+v_{n 1}^{2}\right)\right. \\
& +a v_{n 2}^{4}\left(28 b^{2}-37 b v_{n 1}^{2}-8 v_{n 1}^{4}\right) \\
& \left.+3 v_{n 2}^{6}\left(3 b-2 v_{n 1}^{2}\right)\left(b+v_{n 1}^{2}\right)\right)
\end{aligned}
$$

$$
\begin{aligned}
d_{12}= & -2 \kappa^{-5} a b v_{n 1}^{2} v_{n 2}^{2}\left(4 a^{3} v_{n 1}^{4}+2 a^{2} v_{n 1}^{2} v_{n 2}^{2}\left(4 b-7 v_{n 1}^{2}\right)\right. \\
& +a v_{n 2}^{4}\left(4 b^{2}+15 b v_{n 1}^{2}-16 v_{n 1}^{4}\right) \\
& \left.+v_{n 2}^{6}\left(29 b^{2}-41 b v_{n 1}^{2}+2 v_{n 1}^{4}\right)\right),
\end{aligned}
$$

$$
\begin{aligned}
d_{13}= & 2 \kappa^{-5} a b v_{n 1}^{2} v_{n 2}^{2}\left(-8 a^{3} v_{n 1}^{4}+2 a^{2} v_{n 1}^{2} v_{n 2}^{2}\left(b-7 v_{n 1}^{2}\right)\right. \\
& +a v_{n 2}^{4}\left(b-4 v_{n 1}^{2}\right)\left(10 b+v_{n 1}^{2}\right) \\
& \left.+v_{n 2}^{6}\left(29 b^{2}-41 b v_{n 1}^{2}+2 v_{n 1}^{4}\right)\right),
\end{aligned}
$$




$$
\begin{aligned}
d_{23}= & 2 \kappa^{-5} a b v_{n 2}^{2}\left(4 a^{3} v_{n 1}^{6}-2 a^{2} v_{n 1}^{4} v_{n 2}^{2}\left(4 b+7 v_{n 1}^{2}\right)\right. \\
& -a v_{n 1}^{2} v_{n 2}^{4}\left(10 b^{2}-31 b v_{n 1}^{2}+16 v_{n 1}^{4}\right) \\
& \left.+v_{n 2}^{6}\left(2 b^{3}-9 b^{2} v_{n 1}^{2}-9 b v_{n 1}^{4}+2 v_{n 1}^{6}\right)\right)
\end{aligned}
$$

In equations C-20-C-29, the quantity $\kappa$ is given in equation C-19.

\section{REFERENCES}

Alkhalifah, T., 1998, Acoustic approximations for seismic processing in transversely isotropic media: Geophysics, 63, 623-631, doi: 10.1190/1.1444361.

Alkhalifah, T., 2000a, Prestack phase-shift migration of separate offsets: Geophysics, 65, 1179-1194, doi: 10.1190/1.1444811.

Alkhalifah, T., 2000b, The offset-midpoint traveltime pyramid in transversely isotropic media: Geophysics, 65, 1316-1325, doi: 10.1190/1.1444823.

Alkhalifah, T., 2003, An acoustic wave equation for orthorhombic anisotropy: Geophysics, 68, 1169-1172, doi: 10.1190/1.1598109.

Bakulin, A., V. Grechka, and I. Tsvankin, 2000, Estimation of fracture parameters from reflection seismic data - Part II: Fractured models with orthorhombic symmetry: Geophysics, 65, 1803-1817, doi: 10.1190/1.1444864.

Bender, C. M., and S. A. Orszag, 1978, Advanced mathematical methods for scientists and engineers: McGraw-Hill.

Bleistein, N., and R. A. Handelsman, 1986, Asymptotic expansions of integrals: Dover Publications Inc.

Cheng, J., J. Geng, H. Wang, and Z. Ma, 2011, 3D Kirchhoff prestack time migration in average illumination-azimuth and incident-angle domain for isotropic and vertical transversely isotropic media: Geophysics, 76, no. 1, S15-S27, doi: 10.1190/1.3533913.

Cheng, J., T. Wang, C. Wang, and J. Geng, 2012, Azimuth-preserved local angle-domain prestack time migration in isotropic, vertical transversely isotropic and azimuthally anisotropic media: Geophysics, 77, no. 2, S51-S64, doi: 10.1190/geo2011-0295.1.

Claerbout, J., 1985, Imaging the earth's interior: Blackwell Science Inc.

Farra, V., and I. Psencik, 2013a, Moveout approximations for P- and SV-waves in VTI media: Geophysics, 78, no. 5, WC81-WC92, doi: 10.1190/geo2012-0408.1.

Farra, V., and I. Psencik, 2013b, Moveout approximations for P- and SVwaves in dip-constrained transversely isotropic media: Geophysics, 78 , no. 6, C53-C59, doi: 10.1190/geo2013-0083.1.

Grechka, V., and I. Tsvankin, 1999, 3-D moveout inversion in azimuthally anisotropic media with lateral velocity variation: Theory and a case study: Geophysics, 64, 1202-1218, doi: 10.1190/1.1444627.
Hao, Q., and A. Stovas, 2014, P-wave diffraction and reflection traveltimes for a homogeneous 3D TTI medium: Journal of Seismic Exploration, 23, 405-429.

Hao, Q., and A. Stovas, 2015, The offset-midpoint traveltime pyramid in 2D transversely isotropic media with a tilted symmetry axis: Geophysical Prospecting, 63, 587-596, doi: 10.1111/1365-2478.12201.

Hao, Q., and A. Stovas, 2016a, Analytic calculation of phase and group velocities of $\mathrm{P}$-waves in orthorhombic media: Geophysics, 81, no. 3, C79-C97, doi: 10.1190/geo2015-0156.1.

Hao, Q., and A. Stovas, 2016b, P-wave slowness surface approximation for tilted orthorhombic media: Geophysics, 81, no. 3, C99-C112, doi: 10 .1190/geo2015-0440.1.

Hao, Q., A. Stovas, and T. Alkhalifah, 2015, The offset-midpoint traveltime pyramid in 3D transversely isotropic media with a horizontal symmetry axis: Geophysics, 80, no. 1, T51-T62, doi: 10.1190/geo2013-0322.1.

Schoenberg, M., and K. Helbig, 1997, Orthorhombic media: Modeling elastic wave behavior in a vertically fractured earth: Geophysics, 62, 19541974, doi: 10.1190/1.1444297.

Stovas, A., 2015, Azimuthally dependent kinematic properties of orthorhombic media: Geophysics, 80, no. 6, C107-C122, doi: 10.1190/ geo2015-0288.1.

Stovas, A., and T. Alkhalifah, 2012, A new traveltime approximation for TI media: Geophysics, 77, no. 4, C37-C42, doi: 10.1190/geo2011-0158.1.

Stovas, A., and T. Alkhalifah, 2013, A tilted transversely isotropic slowness surface approximation: Geophysical Prospecting, 61, 568-573, doi: 10 .1111/gpr.2013.61.issue-3.

Sun, X., and S. Z. Sun, 2015, Full-azimuth anisotropic prestack time migration in the local-angle domain and its applications on fracture detection: Geophysics, 80, no. 2, C37-C47, doi: 10.1190/geo2014-0162.1.

Thomsen, L., 1986, Weak elastic anisotropy: Geophysics, 51, 1954-1966, doi: 10.1190/1.1442051.

Tsvankin, I., 1997, Anisotropic parameters and P-wave velocity for orthorhombic media: Geophysics, 62, 1292-1309, doi: 10.1190/1.1444231.

Tsvankin, I., 2001, Seismic signatures and analysis of reflection data in anisotropic medium (1st ed.): Elsevier Science Publishing Company Inc.

Vasconcelos, I., and I. Tsvankin, 2006, Non-hyperbolic moveout inversion of wide-azimuth P-wave data for orthorhombic media: Geophysical Prospecting, 54, 535-552, doi: 10.1111/j.1365-2478.2006.00559.x.

$\mathrm{Xu}, \mathrm{X}$., I. Tsvankin, and A. Pech, 2005, Geometrical spreading of P-waves in horizontally layered, azimuthally anisotropic media: Geophysics, 70, no. 5, D43-D53, doi: 10.1190/1.2052467.

Yan, J., and I. Tsvankin, 2008, AVO-sensitive semblance analysis for wideazimuth data: Geophysics, 73, no. 2, U1-U11, doi: 10.1190/1.2834115.

Yilmaz, O., 2001, Seismic data analysis, processing, inversion and interpretation of seismic data I: SEG.

Yilmaz, O., and J. Claerbout, 1980, Prestack partial migration: Geophysics, 45, 1753-1779, doi: 10.1190/1.1441064. 